\title{
AVERAGES OVER NORMAL MODES OF COUPLED OSCIILATORS WITH APPLICATION TO THEORY OF SPECIFIC HEATS*
}

\section{By ELLIOTT W. MONTROLL (University of Pittsburgh)}

1. Introduction. It is well known that the vibrational contribution to the specific heat at constant volume of a crystalline solid or of a gas composed of complex molecules can be calculated from the Einstein equation ${ }^{1}$

$$
C_{v}=k \sum_{i}\left(h \nu_{i} / 2 k T\right)^{2} / \sinh ^{2}\left(h \nu_{i} / 2 k T\right),
$$

where

$$
\begin{aligned}
h & =\text { Planck's constant } \\
k & =\text { Boltzmann's constant } \\
T & =\text { Temperature in degrees absolute. } \\
\left\{\nu_{i}\right\} & =\text { set of frequencies of normal modes of vibration. }
\end{aligned}
$$

The summation is to be extended over all frequencies. Eq. (1) can also be written as the Stieltjes integral

$$
C_{v}=k \int_{0}^{\nu_{\mathrm{L}}}(h \nu / 2 k T)^{2} d N(\nu) / \sinh ^{2}(h \nu / 2 k T),
$$

where $N(\nu)$ is the number of normal modes with frequencies less than $\nu$; and $\nu_{\mathrm{L}}$ is the largest frequency of a normal mode.

To apply Eqs. (1) or (2) one must either know all the frequencies of normal modes or their distribution function. In the case of complex molecules and crystalline solids composed of $N\left(N=O\left(10^{23}\right)\right.$ in the latter case) particles, the procurement of this knowledge becomes equivalent to the determination of the $(3 N-6)$ normal modes of $N$ coupled oscillators ( 6 being the number of translational and rotational degrees of freedom). Mathematically this means finding the zeroes of a characteristic determinant of order $(3 N-6)$ or the roots of a $(3 N-6)$ th degree equation. In highly symmetrical molecules and crystal lattices this characteristic determinant is factorable into several smaller ones; but to date $N(\nu)$ has never been derived exactly for any molecular model of a real crystal. Numerical approximations have been obtained for $N(\nu)$ but without any estimate of their errors. ${ }^{2}$

By taking traces of powers of the matrix of the determinant mentioned above one can obtain the moments of the distribution function $d N(\nu)$. It is the purpose of this note to show how, without a knowledge of $N(\nu)$ or the frequencies of the individual normal modes, one can obtain by the method of mechanical quadratures averages over the normal modes

* Received Dec. 4, 1946.

1 R. H. Fowler, Statistical mechanics, Comb. Univ. Press, 1936, pp. 86-103.

2 E. W. Kellermann, Phil. Trans. Roy. Soc. 238, 513 (1940); M. Blackman, Proc. Roy. Soc. (A) 148, 365-384 (1935); (A) 159, 417-431 (1937); P. C. Fine, Phys. Rev. 56, 355-359 (1939); M. Iona, Phys. Rev. 60, 822 (1941); E. Montroll, J. Chem. Phys., 10, 218-229 (1942); 11, 481-495 (1943); E. Montroll and D. Peaslee, J. Chem. Phys., 12, 98-106 (1944). 


$$
\bar{f}=\Sigma f\left(\nu_{i}\right)=\int_{0}^{\nu_{\mathbf{L}}} f(\nu) d N(\nu),
$$

(of which (1) is a special case) from the traces of powers of the matrix of the characteristic determinant. An expression will be given for the upper bound of the error in the calculation of (3) from a finite number of moments of $N(\nu)$.

2. Résumé of theory of mechanical quadratures. In this section we shall quote without proof some theorems on mechanical quadratures that will be useful in our work. A detailed exposition of these theorems has been given by G. Szegö. ${ }^{3}$

Let $\alpha(x)$ be a non decreasing function with infinitely many points of increase in the interval $(a, b)$ and suppose the moments

$$
\mu_{n}=\int_{a}^{b} x^{n} d \alpha(x), \quad n=0,1,2, \cdots
$$

exist.

If we orthogonalize the set of non negative powers of $x$, viz. $1, x, x^{2}, \cdots, x^{n}$ with respect to the function $\alpha(x)$ we obtain the set of polynomials $p_{0}(z), p_{1}(x), \cdots$, $p_{n}(x), \cdots$ uniquely determined by the conditions

a) $p_{n}(x)$ is a polynomial of precise degree $n$ in which the coefficient of $x^{n}$ is positive

b) the system $\left\{p_{n}(x)\right\}$ is orthonormal

$$
\int_{a}^{b} p_{n}(x) p_{m}(x) d \alpha(x)=\delta_{m n} \quad m, n=0,1,2, \cdots
$$

Further, for $n \geqq 1$

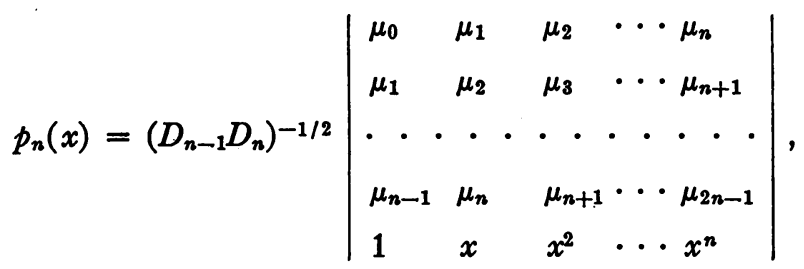

$$
\begin{aligned}
& D_{n}=\left|\begin{array}{cccc}
\mu_{0} & \mu_{1} & \cdots & \mu_{n} \\
\cdot & \cdot & \cdot & \cdot \\
\mu_{n} & \mu_{n+1} & \cdots & \mu_{2 n}
\end{array}\right| \text {. }
\end{aligned}
$$

When $\alpha(x)$ has only a finite number, say $m$, of points of increase there exist only $m$ linearly independent orthonormal polynomials. ${ }^{4}$

Let $x_{1}<x_{2}<x_{3}<\cdots<x_{n}$ denote the zeros of $p_{n}(x)$. There exist numbers $\lambda_{1}, \lambda_{2}, \cdots, \lambda_{n}$ such that by the Gauss-Jacobi mechanical quadrature formula

$$
\int_{a}^{b} F(x) d \alpha(x)=\lambda_{1} F\left(x_{1}\right)+\lambda_{2} F\left(x_{2}\right)+\cdots+\lambda_{n} F\left(x_{n}\right),
$$

where $F(x)$ is an arbitrary polynomial of degree $2 n-1$. The values of the numbers $\lambda$

${ }^{3}$ Szegö, Orthogonal polynomials, Am. Math. Soc. Colloquium Ser., 23, Chapt. 2-3 (1939).

' G. Szegö, loc. cit., 25-27. 
of course depend on $n$. They are often called Christoffel numbers and are given by fhe following equivalent expressions

$$
\begin{aligned}
\lambda_{\nu} & =\int_{a}^{b}\left[\frac{p_{n}(x)}{p_{n}^{\prime}(x)\left(x-x_{v}\right)}\right]^{2} d \alpha(x), \\
\lambda_{\nu} & =\frac{k_{n+1}}{k_{n}} \frac{(-1)}{p_{n+1}\left(x_{v}\right) p_{n}^{\prime}\left(x_{v}\right)}, \\
\lambda_{\nu}^{-1} & =\left\{p_{0}\left(x_{v}\right)\right\}^{2}+\left\{p_{1}\left(x_{v}\right)\right\}^{2}+\cdots+\left\{p_{n}\left(x_{p}\right)\right\}^{2} .
\end{aligned}
$$

Here $k_{n}$ is the coefficient of highest power of $x$ in $p_{n}(x)$ and from (6

$$
k_{n}=\left(D_{n-1} / D_{n}\right)^{1 / 2}
$$

Furthermore, all numbers $\lambda_{\nu}$ are positive, and

$$
\lambda_{1}+\lambda_{2}+\cdots+\lambda_{n}=\alpha(b)-\alpha(a) .
$$

It has been shown by Markoff ${ }^{5}$ that when $F(x)$ is a continuous function with a continuous derivative of order $2 n+1$, (8) can be generalized to

$$
\int_{a}^{b} F(x) d \alpha(x)=\lambda_{1} F\left(x_{1}\right)+\cdots+\lambda_{n} F\left(x_{n}\right)+F^{(2 n)}(\xi) /(2 n) ! k_{n}^{2},
$$

, where $a \leqq \xi \leqq b$. Clearly, if (11) is approximated by (8) an upper bound to the error can be determined by finding the value of $\xi$ for which $F^{(2 n)}(\xi)$ is a maximum and evaluating $F^{(2 n)}(\xi) /(2 n) ! k_{n}^{2}$.

We shall now show that the moments $\mu_{n}$ can be found in a vibration problem if $\alpha(x)$ is interpreted as the number of normal modes with frequencies less than $\nu=x \nu_{L}$ and therefore that (11) can be employed to find averages over all the nomal modes of vibration of the system.

3. Theory of vibrations. When the kinetic and potential energies of a system of coupled elements are given by

$$
\begin{aligned}
& T=\frac{1}{2}\left(a_{11} \dot{q}_{1}^{2}+\cdots+a_{n n} \dot{q}_{n}^{2}\right), \\
& V=\frac{1}{2}\left(b_{11} q_{1}^{2}+\cdots+b_{n n} q_{n}^{2}+2 b_{12} q_{1} q_{2}+2 b_{13} q_{1} q_{3}+\cdots+2 b_{n-1, n} q_{n-1} q_{n}\right),
\end{aligned}
$$

the frequencies $\nu$ of normal modes are given by the roots of the characteristic equation $^{6}$

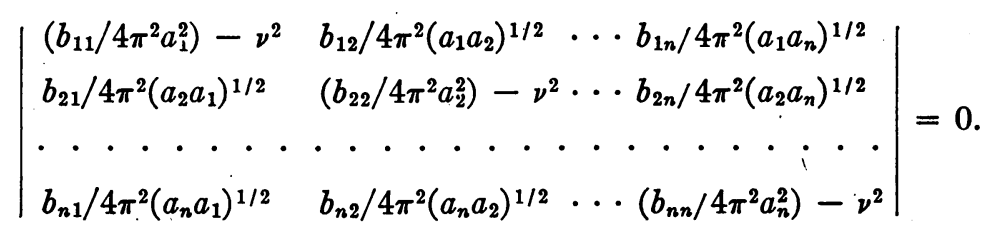

If we let $B$ be the matrix whose elements are $b_{i j} / 4 \pi^{2}\left(a_{i} a_{j}\right)^{1 / 2}$, and $\pm \nu_{1}, \pm \nu_{2}, \cdots, \pm \nu_{n}$ be the $2 n$ roots of (14), and if $\Sigma \nu^{m}$ represents the sum of the $m$ th powers of the roots, then we have

${ }^{5}$ A. A. Markoff, Differenzenrechnung, Leipzig, 1896, p. 29.

- E. T. Whittaker, Analytical dynamics, Camb. Univ. Press, 1927, p. 179. 


$$
\Sigma \nu^{0}=2 n, \quad \Sigma \nu^{2 m+1}=0, \quad \Sigma \nu^{2 m}=2 \text { trace } B^{m} .
$$

Further, if we interpret $x$ in (4) as the ratio $\nu / \nu_{L}$ and $\alpha(x)$ as the number of values of $\nu / \nu_{L}$ (where $\nu$ is a root of (14)) which are less than $x$, it is clear from (4) and (15) that

$$
\begin{aligned}
\mu_{0} & =\int_{-1}^{1} d \alpha(x)=\Sigma \nu^{0}=2 n, \\
\mu_{2 m+1} & =\int_{-1}^{1} x^{2 m+1} d \alpha(x)=\Sigma\left(\nu / \nu_{L}\right)^{2 m+1}=0, \\
\mu_{2 m} & =\int_{-1}^{1} x^{2 m} d \alpha(x)=\Sigma\left(\nu / \nu_{L}\right)^{2 m}=2 \nu_{L}^{-2 m} \operatorname{trace} B^{m} .
\end{aligned}
$$

The averages of interest, (3), are $\bar{f}=\Sigma f\left(\nu_{j}\right)$, where the summation extends over all positive roots of the determinant (14). By defining a new function

and

$$
\begin{array}{lll}
F(\nu)=f(\nu), & \text { if } \quad \nu \geqq 0 \\
F(\nu)=f(-\nu), & \text { if } \quad \nu<0,
\end{array}
$$

(3) can be written as

$$
\bar{f}=\frac{1}{2} \int_{-1}^{1} F\left(x \nu_{\mathrm{L}}\right) d \alpha(x),
$$

which is of the form of (11). Since the moments $\mu_{n}$ can be determined from (16), $p_{n}(x)$ can be evaluated from (6) and $\bar{f}$ obtained by applying (11).

4. Numerical example. To demonstrate the manner of application of (18) let us consider the steps required in the determination of the specific heat of tungsten. It is to be emphasized that the purpose of this example is merely to review the details of a calculation-too few moments are employed to:obtain results that could be expected to be in agreement with observed specific heats.

Tungsten forms a body centered cubic lattice. Its equations of motion have been determined by Fine, and Montroll and Peaslee ${ }^{2}$ who used the assumption that the Cauchy condition on the elastic constants is satisfied. The traces of the characteristic determinant were found in the latter paper and the moments of the frequency distribution were determined. The moments, $\mu_{n}$, there presented in table II differ somewhat from those defined by eq. 4 . Those of table II (when $\tau=2 / 3$ ), which we shall denote by $\mu_{n}^{\prime}$ are related to the $\mu_{n}^{\prime}$ 's of eq. 4 , in a lattice of $N$ atoms, by $\mu_{n}=6 N \mu_{n}^{\prime} / \nu_{L}$, so that the moments $\mu_{0} \cdots \mu_{8}$ are

$$
\begin{array}{lll}
\mu_{0}=6 N, & \mu_{1}=\mu_{3}=\mu_{5}=\cdots=0, & \mu_{2}=\frac{1}{2}(6 N), \\
\mu_{4}=(11 / 36)(6 N), & \mu_{6}=(61 / 288)(6 N), & \mu_{8}=(733 / 4608)(6 N) .
\end{array}
$$

Substituting these moments into (7) we obtain

$$
\begin{gathered}
D_{0}=6 N, \quad D_{1}=\frac{1}{2}(6 N)^{2}, \quad D_{2}=(1 / 36)(6 N)^{3}, \\
D_{3}=\left[65 / 2(36)^{3}\right](6 N)^{4}, \quad D_{4}=\left[(65)(31) / 16(36)^{5}\right](6 N)^{5} .
\end{gathered}
$$

and from (6) 


$$
\begin{gathered}
p_{0}(x)=1 /(6 N)^{1 / 2}, \quad p_{1}(x)=2^{1 / 2} x /(6 N)^{1 / 2}, \quad p_{2}(x)=(3 / 2) 2^{1 / 2}\left(2 x^{2}-1\right) /(6 N)^{1 / 2}, \\
p_{3}(x)=2(2 / 65)^{1 / 2}\left(18 x^{3}-11 x\right) /(6 N)^{1 / 2}, \\
p_{4}(x)=(1 / 4)(2 / 31)^{1 / 2}\left(288 x^{4}-306 x^{2}+65\right) /(6 N)^{1 / 2} .
\end{gathered}
$$

The roots of $p_{4}(x)$ are

$$
x_{1}=-.8769, \quad x_{2}=-.5418, \quad x_{3}=.5418, \quad x_{4}=.8769 .
$$

Thus, from (9c) we see that

$$
\lambda_{1}=\lambda_{4}=(.2172)(6 N), \quad \lambda_{2}=\lambda_{3}=(.2828)(6 N) .
$$

Thus, choosing the function $f(\nu)$ to be

$$
f(\nu)=f\left(x \nu_{L}\right)=k(x \theta)^{2} / \sin ^{2} \theta x,
$$

where $\theta=h \nu_{\mathrm{L}} / 2 k T$ we obtain from (1), (11), and (18

$$
C_{v} \simeq 3 N k\left\{(.4344)(.8769 \theta)^{2} / \sinh ^{2}(.8769 \theta)+(.5656)(.5418 \theta)^{2} / \sinh ^{2}(.5418 \theta)\right\} .
$$

Qualitatively our method is equivalent to replacing the entire frequency spectrum by a small number, say $n$, of specially chosen sharp frequencies. These frequencies and their weight factors are chosen so that the values obtained for all averages over polynomials of degree $(2 n-1)$, or less, are exact. In conclusion, it might be mentioned that in general analogous methods can be used in evaluating averages over characteristic values of linear operators.

\section{THE SUBSONIC FLOW ABOUT A BODY OF REVOLUTION*}

By E. V. LAITONE (Cornell Aeronautical Laboratory)

In cylindrical coordinates the Laplace differential equation, which defines the irrotational incompressible fluid flow, becomes

$$
\phi_{x x}+\phi_{r r}+\frac{1}{r} \phi_{r}+\frac{1}{r^{2}} \phi_{\theta \theta}=0,
$$

where the last term vanishes when the flow has axial symmetry about the $x$ axis.

In this case a solution of Eq. (1) based on a source distribution $f(x)$ per unit length along the $x$ axis from $x=0$ to $x=L$ is

$$
\begin{aligned}
& \phi=u_{\infty} x-\frac{1}{4 \pi} \int_{0}^{L} \frac{f(\xi) d \xi}{\left[(x-\xi)^{2}+r^{2}\right]^{1 / 2}}, \\
& u=\phi_{x}=u_{\infty}+\frac{1}{4 \pi} \int_{0}^{L} \frac{f(\xi)(x-\xi) d \xi}{\left[(x-\xi)^{2}+r^{2}\right]^{3 / 2}}, \\
& v=\phi_{r}=\frac{r}{4 \pi} \int_{0}^{L} \frac{f(\xi) d \xi}{\left[(x-\xi)^{2}+r^{2}\right]^{3 / 2}},
\end{aligned}
$$

where $v / u=(d r / d x)_{0}$ satisfies the fixed boundary conditions given by the body shape.

* Received Dec. 9, 1946. 